\title{
A hierarchy of granular continuum models: Why flowing grains are both sim- ple and complex
}

\author{
Ken Kamrin ${ }^{1, \star}$ \\ ${ }^{1}$ Massachusetts Institute of Technology, Department of Mechanical Engineering, Cambridge, MA 02139
}

\begin{abstract}
Granular materials have a strange propensity to behave as either a complex media or a simple media depending on the precise question being asked. This review paper offers a summary of granular flow rheologies for well-developed or steady-state motion, and seeks to explain this dichotomy through the vast range of complexity intrinsic to these models. A key observation is that to achieve accuracy in predicting flow fields in general geometries, one requires a model that accounts for a number of subtleties, most notably a nonlocal effect to account for cooperativity in the flow as induced by the finite size of grains. On the other hand, forces and tractions that develop on macro-scale, submerged boundaries appear to be minimally affected by grain size and, barring very rapid motions, are well represented by simple rate-independent frictional plasticity models. A major simplification observed in experiments of granular intrusion, which we refer to as the 'resistive force hypothesis' of granular Resistive Force Theory, can be shown to arise directly from rate-independent plasticity. Because such plasticity models have so few parameters, and the major rheological parameter is a dimensionless internal friction coefficient, some of these simplifications can be seen as consequences of scaling.
\end{abstract}

\section{Introduction}

Granular materials have a well-deserved reputation as a complex rheological media [1]. Much debate still exists on what is the constitutive relation for granular flow. Dry granular systems display history- and preparation-dependent strengthening and dilation [2-4], flow anisotropy and normal stress differences [5-7], nonlinear rate-sensitive yielding [8-11], and nonlocality due to the finite size of grains [12-16]. All these phenomena depend sensitively on grain characteristics such as shape and size distribution, frictional properties, and stiffness [10, 17-20]. Continuum models have been proposed of varying complexity to represent these phenomena, with some taking a simpler form while representing fewer effects, and others more complicated forms with the tradeoff of capturing more of the subtleties. Rather than choosing a particular model, the purpose of this review is to highlight the benefits of each model depending on the problem at hand. We will focus on well-developed flow phenomena.

Interestingly, the complexity of granular materials is somewhat problem specific. For motivation, let us compare granular phenomena to a relatively standard material model, say, a linear viscous fluid, whose shear stress $\tau$ and strain-rate $\dot{\gamma}$ are related by $\tau=\eta \dot{\gamma}$ for viscosity $\eta$. In inhomogeneous flow geometries, where the strain-rate field is not uniform, steady granular flow profiles display a characteristic size-effect whereby the size of shear features is influenced directly by the grain size rather than just the

\footnotetext{
^e-mail: kkamrin@mit.edu
}

local homogenized fields, such as stress [10, 21, 22]. In this sense, granular phenomenology is more complex than a viscous fluid, in which the flow rule is fully local; i.e. stress at a position gives flow at that position. Indeed, to obtain predictive stress and flow fields in a granular material, one needs a nonlocal framework to account for flow cooperativity induced by the finite size of grains. However, if the question is reduced, the modeling complexity needed to obtain an adequate solution reduces enormously. For example, consider the problem of determining the resistive force on an arbitrary rigid solid object being dragged through a bed of grains. This problem has direct relevance in granular-solid interactions, such as locomotion in granular media. Here, the quantity being desired is a global net force, rather than spatial fields of flow and/or stress. By reducing the question in this way, it turns out that very simple models provide an accurate solution. In particular, reduced-order resistive force models from viscous flow theory can be translated to the granular problem, and often work better than in the original viscous fluid application [23-25]. It would seem that in these kinds of problems, interestingly, granular media may behave simpler than a viscous fluid.

Herein, we discuss the behaviors of granular media in terms of a nested family of continuum models. The basic features of granular flows can be predicted with simple frictional plastic models containing only a single (dimensionless) internal friction coefficient [26-28]. However, obtaining details at the next order of precision often incurs a rather large leap in modeling complexity, although certain specific observables are affected less than others by 
which model is used. We discuss the Nonlocal Granular Fluidity (NGF) model as an example of a more complex model [12-14, 29], which augments the frictional plastic form with both rate-dependence and particle size dependence. We begin by describing the continuum models at hand, and then follow up with demonstrations focusing on weighing the need for simpler models, which are more amenable to analytical tools, with the need for more detailed forms that have greater field accuracy but at a larger computational cost.

\section{Nested Family of Flow Models}

The simplest continuum models for granular flow date back to the ideas of Coulomb in the 1700's. They are based on the concept of a frictional yield criterion, where the shear stress needed to achieve plastic flow grows linearly with the applied pressure. These notions have been extended to three-dimensions by Mohr and further simplified by Drucker and Prager [30] where they are common tools in geotechnical engineering [26, 31].

To write a steady flow model based on these principles, we first assume a standard momentum balance

$$
\rho \dot{\mathbf{v}}=\nabla \cdot \sigma+\mathbf{b}
$$

where $\sigma$ is the Cauchy stress, $\rho$ is the mass density, $\mathbf{v}$ is the velocity, $\nabla$ is the spatial gradient operator, and $\mathbf{b}$ is a body force (typically the gravitational body force).

For the sake of concreteness and simplicity, let us make a rigid-plastic assumption, neglecting the elastic response of the grains to approximate all flow as plastic flow. The constitutive response can now be expressed as constraints on the stress in terms of flow and any relevant state variables.

Let us define the (hydrostatic) pressure $p=-\sigma_{i i} / 3$ and equivalent shear stress $\tau=\sqrt{\sigma_{i j}^{\prime} \sigma_{i j}^{\prime} / 2}$ where ' denotes the deviator, i.e. $\sigma^{\prime} \equiv \sigma+p \mathbf{I}$ for $\mathbf{I}$ the identity tensor. Further, we define the strain-rate tensor by $\mathbf{D}=\left(\nabla \mathbf{v}+\nabla \mathbf{v}^{T}\right) / 2$ and the equivalent shear-rate by $\dot{\gamma}=\sqrt{2 D_{i j}^{\prime} D_{i j}^{\prime}}$. It is reasonable to assume a roughly incompressible response since dilation/contraction should cease at the steady state, allowing us to write $\operatorname{trD}=0$. We note that certain secondary flow phenomena may be missed by neglecting dilatation [32]. We can also make the assumption of codirectionality of the stress and strain-rate tensors, which when combined with incompressibility gives

$$
\mathbf{D}=\lambda \sigma^{\prime}
$$

where $\lambda$ is a non-negative scalar multiplier. This assumption neglects the presence of anisotropy, which causes the eigenvectors of stress and strain-rate to misalign $[6,7,9]$, as well as normal stress differences [5, 33], which cause the eigenvalues of strain-rate to differ by more than a single scalar factor from those of the deviatoric stress. Both these effects are known to exist; misalignment can be upwards of five degrees and normal stress differences in the range of ten percent are known to occur.
We now summarize three granular flow models, each adding more complexity to the one before it.

Frictional plasticity: In the well-developed limit, transient effects cease and we can express the Drucker-Prager failure criterion as

$$
\mu \equiv \tau / p=\mu_{s} \text { if } \dot{\gamma}>0 \text {, and } \tau \leq \mu_{s} p \text { otherwise. }
$$

Here, $\mu_{s}$ is an internal friction coefficient, deemed constant in this model. The above scalar relation combined with codirectionality and momentum balance provides a closed system of equations. Note this is a non-associated plasticity model. It should also be clarified that because we consider cohesionless grains, we must supply additional physics to ensure the pressure stays non-negative, i.e. no tension. We can achieve this by requiring

$$
p=0 \text { if } \rho<\rho_{c}
$$

where $\rho_{c}$ is a critical packing fraction needed for the grains to just barely be in contact [27, 28]. If grains open up beyond this point, the system is modeled as disconnected and thus stress-free, as in a low-temperature granular gas. Note that this type of opening is distinct from plastic dilation in which grains dilate but still support a non-zero pressure. Numerically, the opening criterion can be implemented by reintroducing the elastic part of the deformation and requiring the elastic moduli to vanish when $\rho<\rho_{c}$. The constitutive model comprised of a Drucker-Prager failure criterion, codirectionality, incompressibility, and the opening constraint will be referred to as frictional plasticity.

Inertial rheology: When the inertial number, $I \equiv \dot{\gamma} \sqrt{\frac{\rho_{s} d^{2}}{P}}$, becomes large enough, typically $I>10^{-1}$, the effect of rate on the plastic flow becomes noticeable. Here, $d$ is the (mean) grain size and $\rho_{s}$ is the solid density of the particle material. Rate effects in granular flow have been observed back to Bagnold in the 1950's [8] but were formalized more generally in the early to mid 2000's [9, 10]. The key notion is that the rate influences the plastic flow rule by causing the internal friction coefficient to depend on $I$. Most data (but not all, [34]) shows it grows with $I$. A common fit form [35] is

$$
\mu=\hat{\mu}(I)=\mu_{s}+\Delta \mu /(b I / \Delta \mu+1)
$$

where $\mu_{s}$ is now the static friction coefficient, approached as $I \rightarrow 0$. Below $\mu_{s}$ no plastic flow can occur. $\Delta \mu$ and $b$ are dimensionless material constants describing how $\mu$ increases with $I$.

It is useful to consider circumstances that induce nonnegligible rate effects. Because $I$ grows with strain-rate but decreases with pressure, inertial effects matter most where zones near free surfaces are flowing quickly. In fact, the clearest example of rate-sensitivity can be observed in flows of granular layers down inclined planes. A tall layer of grains on a plane tilted upward by $\theta$ experiences $\mu=\tan (\theta)$ everywhere. According to $\mathrm{Eq} 5$, as verified in 
simulations [22, 33], steady flows down the incline occur for $\mu_{s}<\tan (\theta)<\mu_{s}+\Delta \mu$ with the flow rate increasing as $\theta$ increases. Without the dependence on $I$, one would predict that steady flow only occurs at $\tan \theta=\mu_{s}$ and above this angle, the grains accelerate without reaching a steady profile. The finite range of angles that admit steady flows down an incline exemplify the correction for rate presupposed by the inertial rheology.

Nonlocal Granular Fluidity: In a number of circumstances, it is found that the inertial rheology is still insufficient to describe observed flow profiles. Flows where the strain-rate field is inhomogeneous often have wider flow features than would be predicted by the inertial rheology. Counter to the $\mu_{s}$ yield criterion supposed by the inertial rheology, it is found that if part of the granular media is flowing, adjacent parts, even those with $\mu<\mu_{s}$, are also flowing even if just very slowly [21, 36, 37]. Moreover, regarding flows down inclines, it is observed that thinner layers of grains require higher $\theta$ to stop flow compared to thicker systems, even though both obey $\mu=\tan \theta[22,38]$. The explicit dependence of strength on system size goes beyond local mechanics. Other examples of behaviors that suggest an intrinsic length-scale must be introduced include the so-called secondary rheology of granular media [39-41], wherein flow induced at one point causes non-trivial changes to the rheology observed elsewhere. The effect decays away from the point of induced motion, scaled by the grain size.

What these phenomena have in common is that they suggest cooperativity of the grain motion over space must be represented within the rheology. The natural size-scale for this cooperativity is the size of the grains themselves, $d$. There are a number of general modeling frameworks in the literature that allow the inclusion of an intrinsic lengthscale. These include micropolar or Cosserat models that introduce a couple stress field [15], gradient plasticity theories that permit power expenditure over strain gradients [42], thermal models that introduce a diffusing temperature variable (including specialized forms such as Granular Solid Hydrodynamics) [43, 44], and partial fluidization models that assume a diffusing order parameter governing the amount of fluid vs solid constitutive behavior at a point $[16,45]$. Some of these models invoke assumptions that change the expression of momentum balance, Eq 1, while others leave momentum balance unchanged while introducing new field equations that must also be simultaneously solved. The model we will focus on, the NGF model, is of the latter type. It began as a granular extension to existing fluidity frameworks for emulsion flow [46, 47] but has recently been reconciled secondarily through alternative means [48].

The model is based on the introduction of a new scalar state field called the 'granular fluidity' denoted $g(\mathbf{x})$, which is assumed to exist throughout the body of material. Recent work has helped to establish the nature of the $g$ field as a kinematically observable state variable [49]. Its dynamics are assumed to obey a reaction-diffusion form analo- gous to a non-standard Landau-type equation:

$$
t_{0} \dot{g}=A^{2} d^{2} \nabla^{2} g-\Delta \mu\left(\frac{\mu_{s}-\mu}{\mu_{2}-\mu}\right) g-b \sqrt{\frac{\rho_{s} d^{2}}{P}} \mu g^{2}
$$

Here, $t_{0}$ is a constant time-scale and $\mu_{2} \equiv \mu_{s}+\Delta \mu$. The constitutive relation then calls upon the $g$ field to relate stress and strain-rate, i.e.

$$
\mu=\dot{\gamma} / g
$$

Because of the $A^{2} d^{2} \nabla^{2} g$ term in the evolution of $g$, the spatial variation of the fluidity field is influenced directly by the mean particle size. This then causes the relation between stress and strain-rate at a point to depend on behavior not localized to that point, but influenced by nearby events. If the nonlocal amplitude $A$ is set to 0 , the steady solutions of the system reduce identically to the inertial rheology. $A$ is the only new material parameter, beyond those of the inertial rheology. It is dimensionless and order one; $A \approx 0.5$ for glass beads and 0.75 for disks $[12,13,17]$. One could view Eq 6 like a Landau-type form where the last two terms behave like an 'energy derivative' of an energy function with a minimum at $g=0$ if $\mu \leq \mu_{s}$ and a non-zero minimum if $\mu>\mu_{s}$ [48]. As shall be seen in the next section, the model above is able to reconcile multiple manifestations of nonlocality in granular flows. Note that the difference between all three models can be seen in how each one relates $\mu$ to the other fields; compare Eqs 3, 5 , and 7 .

Equation 6 can be approximated to a steady-state-only form by performing linearization [48] to yield

$$
g=g_{l o c}(\mu, p)+\xi(\mu)^{2} \nabla^{2} g
$$

where $g_{l o c}$ is what the fluidity would be if the inertial rheology were correct, and $\xi$ is a dynamic correlation length. These take the form:

$$
\begin{aligned}
g_{l o c}(\mu, p) & =\max \left(0, \frac{1}{\mu} \sqrt{\frac{P}{\rho_{s} d^{2}}} \hat{\mu}^{-1}(\mu)\right) \\
\xi(\mu) & =A d \sqrt{\frac{\mu_{2}-\mu}{\Delta \mu\left|\mu-\mu_{s}\right|}} .
\end{aligned}
$$

where $\hat{\mu}$ comes from Eq 5. Equation 8 allows us to see that the fluidity is a sum of two influences. The term $g_{l o c}$ indicates the effect of local stress on the fluidity; it vanishes wherever $\mu<\mu_{s}$. The second term indicates that disturbances from neighboring media can also influence the fluidity. The function $\xi(\mu)$ diverges as $\mu \rightarrow \mu_{s}$ on both sides, which has been verified in numerical simulations [12] and comparisons with experimental data [13]. This bears similarities to diverging length-scales in phase transitions.

To provide intuition for how the NGF model works, suppose the stress is large enough to cause $g_{l o c}>0$ at some position. The above relation indicates that this region acts like a fluidity source, from which fluidity can then diffuse into neighboring zones, even those for which $g_{l o c}$ is zero, allowing material to flow in regions where local relations would predict no flow. Regarding boundary 
conditions for $g$, two common choices used are $d g / d n=0$ for $n$ the outward normal to the surface, and $g=g_{l o c}$. Both cause minimal 'disruption' at boundaries $[12,13]$. As described mechanically in [48], the Neumann boundary condition can be seen as a sort of conduction-free condition that prevents the fluidity field from expending power at the boundary. The choice of which boundary condition to use matters less in large geometries than in thin ones; in the case of thin geometries, precise $g$ boundary conditions can be inferred from existing data, as done in [14].

\section{Nonlocal Granular Fluidity Model}

The most obvious indication of nonlocal effects in steady flows is if the $\hat{\mu}(I)$ relation, obtained in planar shear tests, breaks down in flows that are inhomogeneous. Suppose for example an annular shear flow (Fig 1(a)). Locally, the flow resembles planar simple shearing. Each element of material, at steady-state, experiences a steady packing fraction and a steady stress state (modulo rotation). Absent a nonlocal effect, one would conclude these fields should obey $\hat{\mu}(I)$ since each material element experiences the same set of conditions that an equivalent planar shear flow experiences, and planar flows strongly confirm the inertial rheology at steady state. However, this is not the case.

In [21] a number of 2D simulations of annular shear were performed. At steady state, values of $\mu$ and $I$ were coarse-grained at many locations and scatter-plotted. This was done for several values of inner wall speed $v_{\text {wall }}$ and several values of $R / d$, see Fig 1(b-c). It was observed that, primarily for low $I$, the locus of $\mu$ and $I$ was not one-toone. Zones below $\mu_{s}$ were observed to flow. The inertial rheology, calculated from simple shear tests, can be seen to emerge in the limit $R / d \rightarrow \infty$ (stars in Fig 1(b)) and wherever $I$ is sufficiently large. In Fig 1(c), zones flowing under $\mu_{s}$ display a curious rate-independence; the three data sets corresponding to the slowest wall speeds have essentially the same range of $\mu$ but have $I$ ranges differing by orders of magnitude. The solid lines in the figure show predictions of the steady-state-only approximation of the NGF model. It is observed that all modes of branching of these different curves are correctly captured by the NGF model without any recalibration [12]. The NGF model captures the return to inertial behavior in the large $R / d$ limit and large $I$ limit because in these cases the nonlocal term $\xi^{2} \nabla^{2} g$ is dwarfed by $g_{l o c}$ in Eq 8 . The NGF model proved the ability to predict flow fields in these geometries over several orders of magnitude, well into the slow creeping zones, where inertial rheology alone fails.

The NGF model was then implemented in 3D [13]. This was done using a customized finite-element routine in Abaqus [50]. We first modeled the split-bottom cell geometry (Fig 2(a)), a flow environment that serves as a benchmark for flow rheologies, as no previous continuum model was able to correctly predict its flow fields. To compare against actual experiments, which used glass beads, the inertial law parameters, $\mu_{s}, \Delta \mu$ and $b$, were chosen to match previously published values for beads, and $A$ was chosen

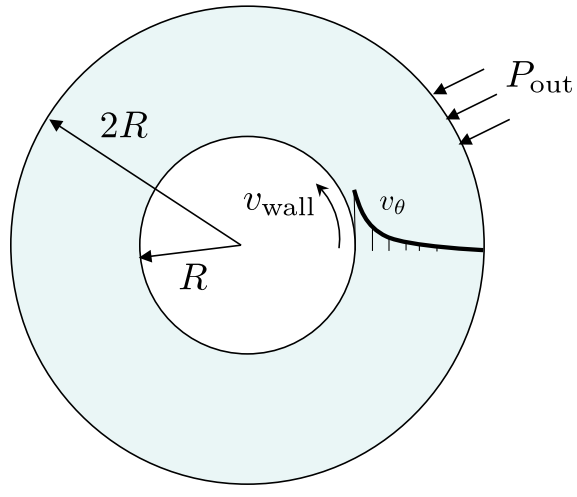

(a)

(b)

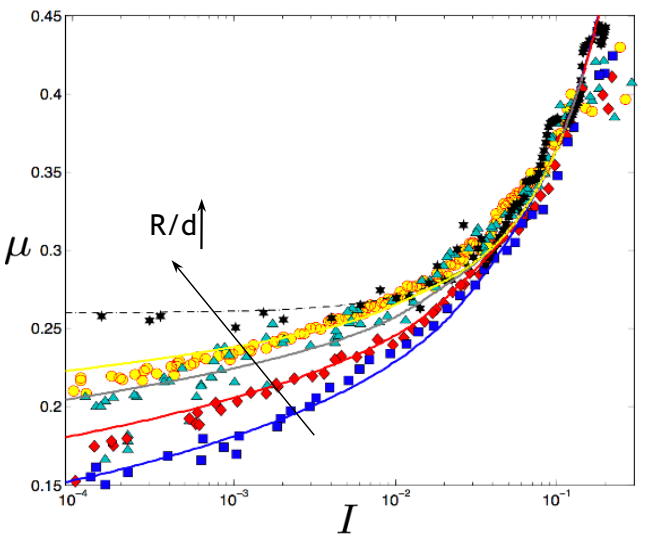

(c)

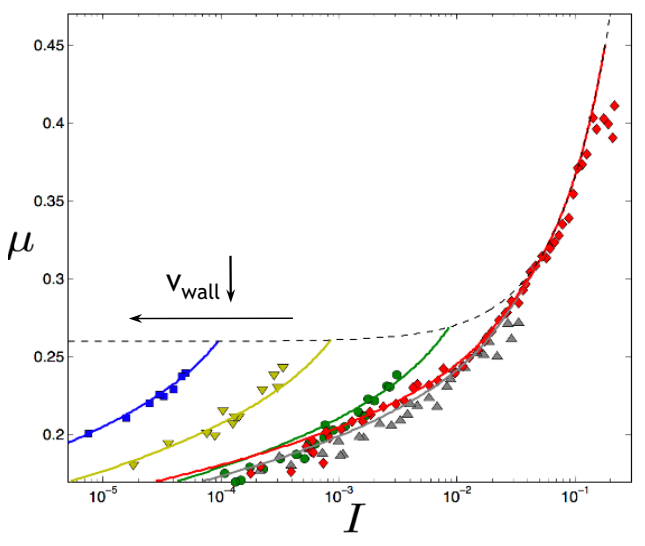

Figure 1: (a) Setup of the annular shear geometry. (b-c) Locus of $\mu$ vs $I$ when geometry size relative to grain size is varied with wall speed held fixed (b), and when wall speed is varied with geometry fixed (c). Symbols are results from DEM simulations, dashed line is the prediction of the inertial rheology for all cases, and solid lines are predictions of the NGF model. Some images/data taken from [12].

at 0.48 . Note that the inertial rheology on its own has been shown insufficient to describe these flows [51, 52].

When a split bottom cell achieves steady state the flow field at the top surface displays a wide shear band, as can be seen in plots of the normalized revolution rate $\omega(r)=v_{\theta}(r) / \Omega r$, where $v_{\theta}$ is the grain speed (which is all 
(a)

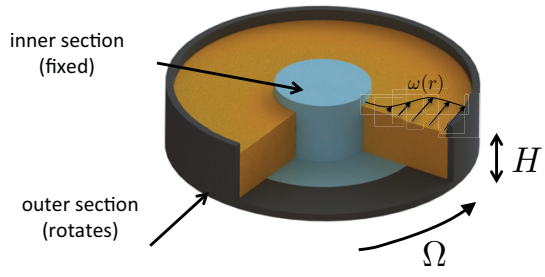

(b)

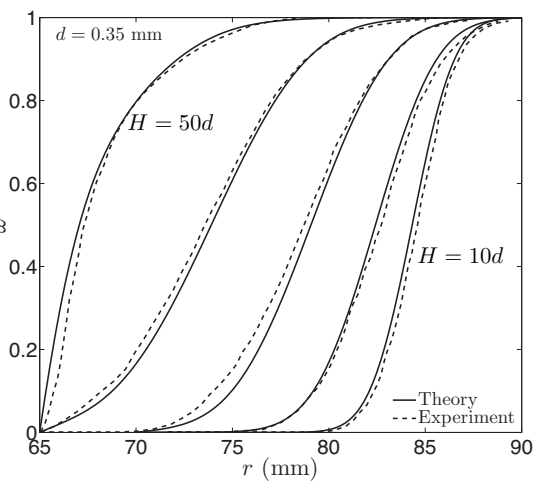

(c)

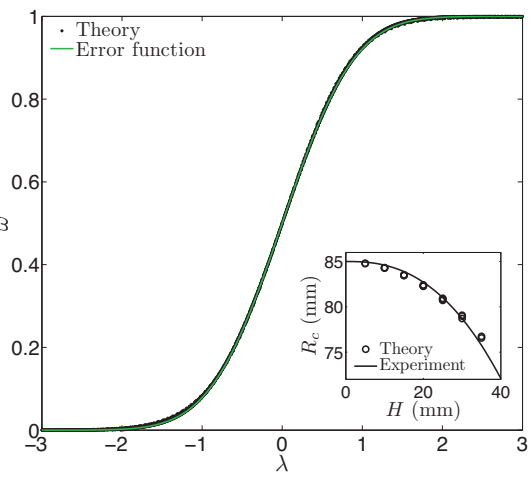

(d)

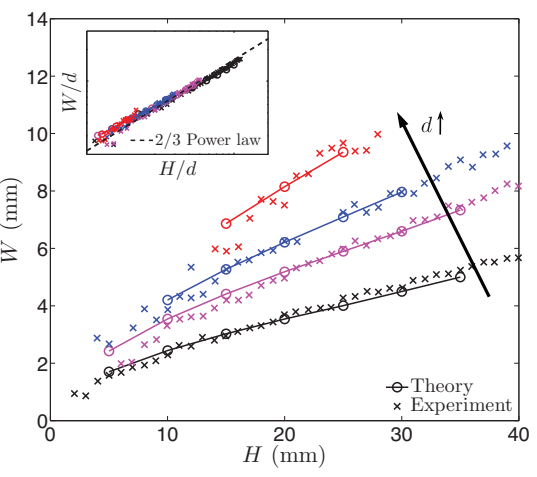

Figure 2: (a) Setup of the split-bottom cell. Grains fill an annular trough whose bottom is split along a circle so that the outer piece can rotate with respect to the inner. (b) Theory vs experiment for the flow field on the free surface as filling height increases. (c) In 22 NGF simulations of shallow flows for various $H$ and $d$ (black points), the topsurface flow profile collapses to an error function shape (green line). (d) Theory vs experiment for width of the shear-band on the top surface in terms of mean grain size and filling height. Some images/data taken from [13].

in the $\theta$ direction). The band is couched in the interior as long as $H$ is small enough; for deeper layers, the shear band hits the inner wall. Predictions of the model vs the
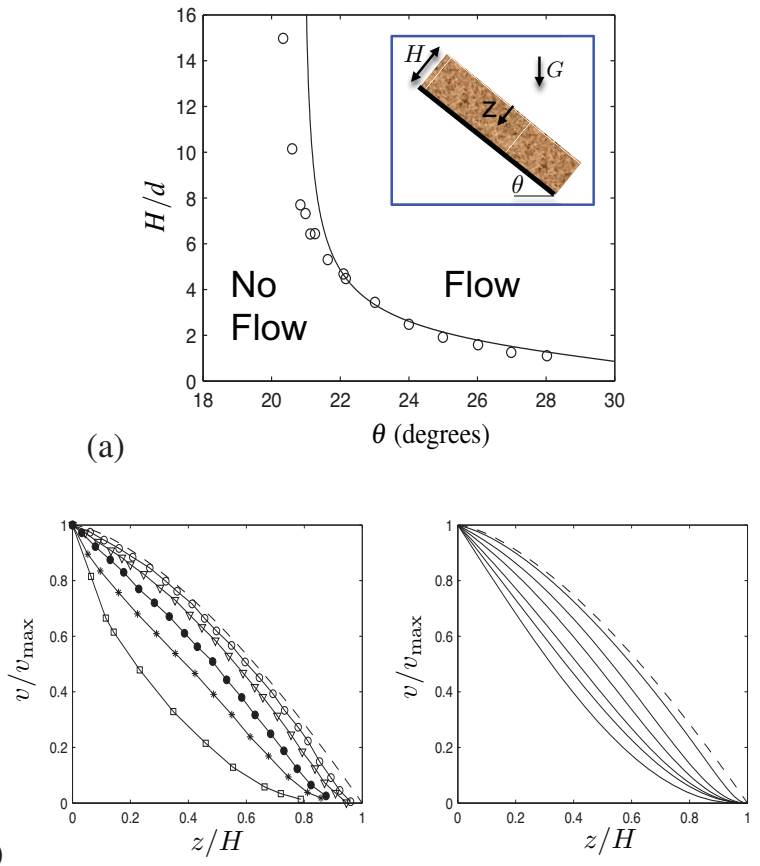

Figure 3: (a) Predicted (line) vs experimental (circles) $H_{\text {stop }}$ values. Flow geometry shown in inset. $G$ is gravitational acceleration. (b) [Left] Flow profiles normalized by $v_{\max }=v(z=0)$ as given from discrete particle simulations. $H / d$ increases from bottom to top with fixed inclination. [Right] Theoretical result using NGF. Dashed line is the Bagnold profile, approached at the largest $H$ in both cases. Some data/images taken from [14].

experimental results for five values of $H$ can be seen in Fig 2(b). The model correctly spreads the shear band and moves it inward as filling height increases.

One amazing fact about $\omega(r)$ is that it is observed experimentally, for various shallow $H$ values, to have a universal shape. Letting $R_{c}$ denote the center of the shear band and $W$ the width, all such flows can be expressed with remarkable precision by an error function [36]: $\omega=$ $\operatorname{erf}\left(\frac{r-R_{c}}{W}\right)$. Interestingly, this collapse to an error function is equally present in solutions to the NGF model. Figure 2(c) shows the error function collapse for 22 combinations of $d$ and $H$, each test contributing a set of black points in the figure. The model's prediction of where the shearband center is, $R_{c}$, is also captured (see inset). Lastly, it should be noted that the split-bottom geometry forces a model to be able to disentangle the coupled effects of the micro-scale $d$ and the geometric scale $H$, in determining the width of the shear-band at the top surface $W$. Figure 2(d) compares experiments to theory for many $H$ and $d$ values and it can be seen that the model consistently predicts the correct shear-band width.

As noted previously, there are multiple incarnations of grain size effects in granular media, beyond their influence on steady flow profiles. Another major size-effect is the so-called $H_{\text {stop }}$ phenomenon. In flow down long inclined planes (see Fig 3(a) inset), $\mu$ is uniform throughout and 


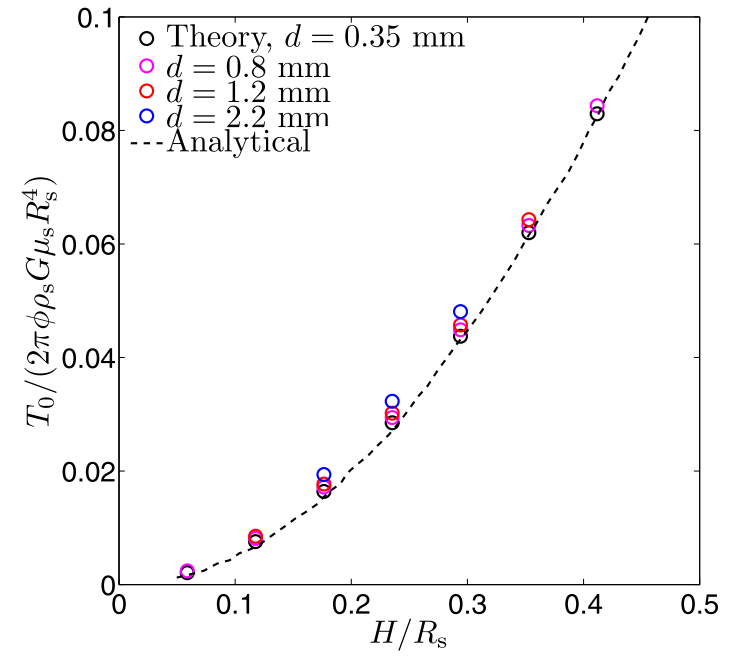

Figure 4: Total torque $T_{0}$ on the outer wall of a splitbottom cell. Symbols are solutions of NGF model for various particle sizes, line is an analytical form assuming an infinitely thin shear band [52]. $R_{s}$ is the split radius, $\Phi$ is the packing fraction. Some data and/or image content taken from [13].

given by $\tan \theta$. The inertial rheology would predict, thus, that only $\theta$ determines if the layer will flow. However, experiments and particle simulations show that whether or not a flowing layer stops depends also on relative filling height, $H / d[22,38]$. The function $H_{\text {stop }}(\theta)$ marks the phase boundary between flowing and static inclined layers, i.e. the locus of points where a flowing system comes to a stop.

Looking at Eq 6 in this geometry, using homogeneous boundary conditions for $g$ implied by existing data [22], we can perform a stability analysis about the global noflow solution, $g=0$, to determine when a static state is the stable solution. The answer gives a prediction for the $H_{\text {stop }}$ curve, which is shown against experimental data for glass beads in Fig 3(a) [14]. Note that the model uses all the same material parameters as the ones used to predict split-bottom flows, though this is answering a very different question - here we are asking whether flow should happen at all, whereas previously we were concerned with the spatial flow profile of grains in a driven process. That said, when flow does occur on the incline, the NGF model still succeeds in predicting its profile. In fact, when the filling height is not much larger than $H_{\text {stop }}$, the flows still show a marked difference from the 'Bagnold profile' predicted by the inertial rheology. As filling height increases, such that $H / d$ is sufficiently large, the Bagnold flow profile re-emerges as the solution. This classical flow profile can also be obtained from the simple scaling of pressure with strain-rate squared when packing fraction is constant [53], which is largely the case in inclined plane flows. In view of Fig 3(b), it can be seen that the qualitative change in the shape of the flow profile as $H$ grows above $H_{\text {stop }}$ is well-captured by the NGF model.

\section{Rate-Independent Frictional Plasticity}

To understand circumstances where the machinery of the nonlocal model may not be necessary, consider the data in Fig 4. This shows the total normalized torque needed to rotate the outer wall of a split-bottom cell, comparing the NGF model for various grain sizes to an analytical result assuming an infinitely thin shear band [52]. Interestingly, the grain size has only a small effect on the torque. Although the size-effect within the NGF model can have a significant effect on the granular flow field, force-like quantities on boundaries appear to be minimally affected by the corrections due to particle size. Further, as noted previously, rate effects due to large $I$ are most significant near free surfaces in fast flow. Taken together, we hypothesize that the problem of determining forces on macro-scale submerged boundaries may be modeled well with the simplest granular rheology, frictional plasticity.

This idea can be checked by comparing data on forces on buried plates moving in various ways in a granular bed. In [23], as shown schematically in Fig 5(a), a small plate buried in the grains $(0.3 \mathrm{~mm}$ beads) was angled in various directions $\gamma$ and moved in various directions $\beta$. In each case the resistive force $\left(F_{x}, F_{z}\right)$ of the grains acting on the plate is measured. It is convenient to scale the measured forces by plate depth times plate area, yielding resistive force coefficients, denoted by $\left(\alpha_{x}, \alpha_{z}\right)$. Figure 5(b), left column, shows experimentally obtained plots of $\alpha_{x}$ and $\alpha_{z}$. We have run finite-element simulations of rough plates moving through frictional plastic continuous media to attempt to model these experiments [28]. The parameters $\rho_{s}$ and $\mu_{s}$ were selected using the density and angle of repose of the same grains as the experiments, as reported in [23]. The corresponding plots of resistive force coefficients are shown in the right column of Fig 5(b). A striking similarity, both in shape and scale can be seen between the experiment and model. It should be noted, that these demonstrations all utilize intruders that are sufficiently large compared to $d$ and moving at a rate that makes $I$ small enough to be neglected. Should either of these conditions be removed, it is unclear how strong the collapse would be.

In the study of resistive forces on generally-shaped intruding objects, a strange experimental correlation has been made, which we call the resistive force hypothesis $[23,24,54]$. It states that the stress acting on an arbitrary surface element of the intruding object can be computed by assuming it is an isolated plate and using the empirical relations from Fig 5(b) to approximate the local resistance. The experimental accuracy of this immense simplification is surprising since it presupposes that the stress acting on a small part of the intruding object is independent of the geometry and motion of the rest of the object; a complete lack of cross-correlation. Moreover, the accuracy of this hypothesis in grains is actually higher than the analogous local drag models developed for viscous fluids [55] — this is particularly ironic since the granular version was inspired by the fluid one, which dates back to the 1950's [56]. Since its first demonstration, it was unclear why the granular resistive force hypothesis works so well. However, as shown in Fig 6, this strange simplifi- 


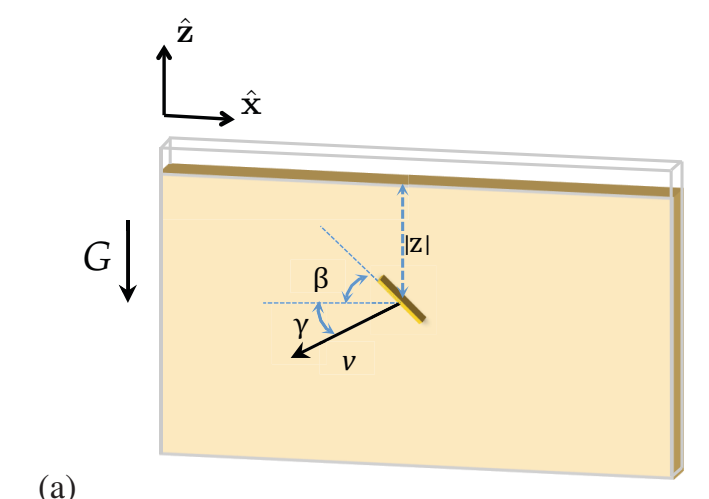

(a)

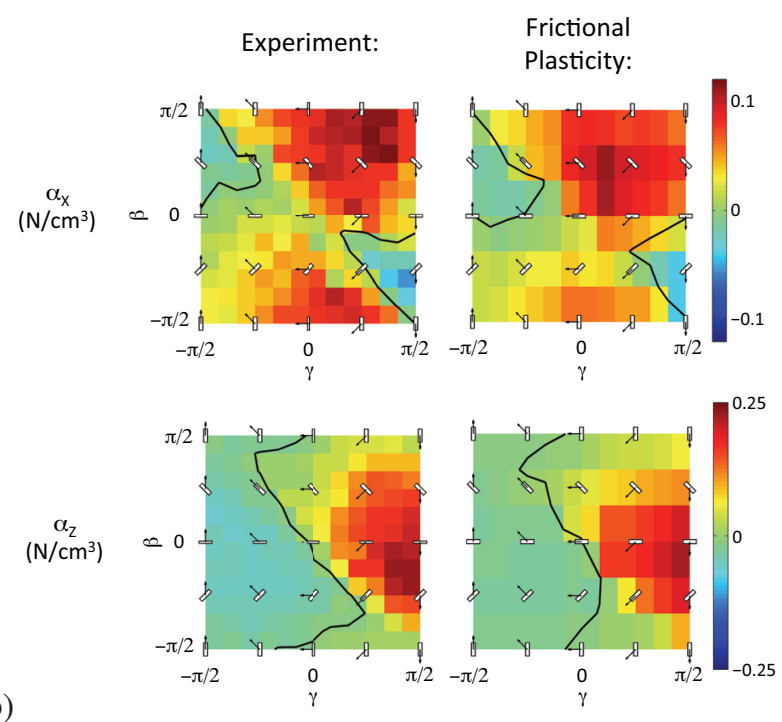

Figure 5: (a) Experimental schematic of submerged plate drag tests. At a prescribed depth $|z|$, the angle of intrusion $\gamma$ given by the imposed velocity $\mathbf{v}$ is varied, as well as the tilt angle $\beta$. (b) The components of the resistive force per depth per area of the experiments compared against the corresponding results from frictional plasticity simulations. The experimentally determined repose angle and grain density were used to calibrate the frictional plasticity model. Images modified from [28].

cation comes out in direct simulations of frictional plasticity. The figure shows, in blue, the force distributions arising from frictional plasticity simulations of a square cross-section intruder and a circular cross-section intruder. In red are the forces distributions implied by supposing Fig 5 can be applied on all surface elements of the leading surface of the objects. The distributions are very close, and, when summed to produce a resultant, the two agree almost exactly (resultant forces shown at the center of the objects). Further demonstrations of the resistive force hypothesis emerging in simulations of frictional plasticity can be found in [28].

A particular benefit of frictional plasticity is that it depends on very few input parameters, just $\rho$ and $\mu_{s}$. As a result, certain analytical tools are an accessible option
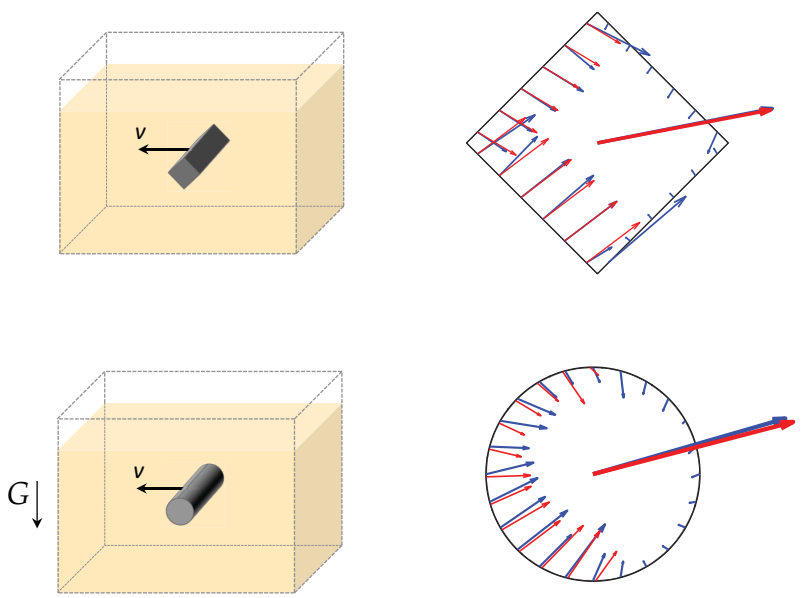

Figure 6: Circular and square cross-section intruders are simulated moving leftward in frictional plastic media, producing the force distribution shown with blue arrows. The red arrows are the force distribution arising from the resistive force hypothesis, obtained by assigning forces consistent with Fig 5, which were themselves obtained from dragging small flat plates. Some images/data taken from [28].

that would otherwise be too difficult to use in more complex models. For example, dimensional analysis can be used to understand various behaviors of the frictional plastic flow rule. We have shown using dimensional analysis why, in certain cases, the resistive force hypothesis is so accurate. For example, dimensional analysis allows us to show, without solving any differential equations, that the total force on a large square 'garden hoe' with its top edge flush with the granular free surface must be exactly equal to the prediction made under the resistive force hypothesis [28]. We are currently studying the potential uses of scaling laws derivable from frictional plasticity in applications like granular locomotion [57].

With regard to the fact that the resistive force hypothesis emerges more strongly in frictional plasticity than in viscous fluid, consider the following. The shear behavior in viscous media is given by a dimensional parameter (viscosity) whereas it is given by a dimensionless parameter in frictional media $\left(\mu_{s}\right)$. Because viscosity has dimensions, the result is that the Reynolds number for fluids is a mixture of viscosity and system-defined length and velocity scales. On the other hand, for frictional plasticity, the equivalent dimensionless number is simply $\mu_{s}$. Because no system-wide lengths or velocities are needed to build this characteristic dimensionless group, a number of simplifications arise in the scaling analysis of frictional systems compared to viscous ones. For example, from scaling alone, the same garden hoe test in a viscous fluid can be shown inconsistent with the resistive force hypothesis [28]. 


\section{Conclusion}

Granular flow models have a wide assortment of complexity. Here we have emphasized that the degree of complexity needed to capture certain phenomena varies dramatically and sensitively on the specific fields of interest and relative size of the geometry. The behavior of the granular velocity field shows subtle dependence on particle size, which influences the flow distribution in inhomogeneous flow geometries, and can cause jamming in smaller geometries even when local stresses would seem to be large enough to induce flow. However, global forces on macroscale moving boundaries can be modeled well using basic frictional plasticity. It could be said then, that the simultaneous simplicity and complexity of different granular flow observables can be attributed to the immense simplification of going from those observables for which NGF is needed to those where frictional plasticity suffices.

\section{References}

[1] J.D. Goddard, Applied Mechanics Reviews 66, 050801 (2014)

[2] A. Schofield, P. Wroth (1968)

[3] D.M. Wood, Soil behaviour and critical state soil mechanics (Cambridge university press, 1990)

[4] S. Roux, F. Radjai, in Physics of dry granular media (Springer, 1998), pp. 229-236

[5] M. Depken, J.B. Lechman, M. van Hecke, W. van Saarloos, G.S. Grest, Europhysical Letters 78, 58001 (2007)

[6] T. Weinhart, R. Hartkamp, A.R. Thornton, S. Luding, Physics of Fluids (1994-present) 25, 070605 (2013)

[7] L. S, Particulate Science and Technology 26, 33 (2008)

[8] R.A. Bagnold, Proceedings of the Royal Society of London Series A 225 (1954)

[9] F. da Cruz, S. Emam, M. Prochnow, J.N. Roux, F. Chevoir, Physical Review E 72, 021309 (2005)

[10] G. MiDi, The European Physical Journal E 14, 341 (2004)

[11] P. Jop, Y. Forterre, O. Pouliquen, Nature 441, 727 (2006)

[12] K. Kamrin, G. Koval, Physical Review Letters 108, 178301 (2012)

[13] D.L. Henann, K. Kamrin, Proceedings of the National Academy of Sciences 110, 6730 (2013)

[14] K. Kamrin, D.L. Henann, Soft matter 11, 179 (2015)

[15] L. S. Mohan, K. K. Rao, P.R. Nott, Journal of Fluid Mechanics 457, 377 (2002)

[16] I.S. Aranson, L.S. Tsimring, Physical Review E 65, 061303 (2002)

[17] K. Kamrin, G. Koval, Computational Particle Mechanics 1, 169 (2014)

[18] E. Azéma, F. Radjai, G. Saussine, Mechanics of Materials 41, 729 (2009)

[19] E. Azéma, N. Estrada, F. Radjai, Physical Review E 86, 041301 (2012)
[20] P.G. Rognon, J.N. Roux, M. Naaïm, F. Chevoir, Physics of Fluids (1994-present) 19, 058101 (2007)

[21] G. Koval, J.N. Roux, A. Corfdir, F. Chevoir, Physical Review E 79, 021306 (2009)

[22] L.E. Silbert, J.W. Landry, G.S. Grest, Physics of Fluids (1994-present) 15, 1 (2003)

[23] C. Li, T. Zhang, D.I. Goldman, Science 339, 1408 (2013)

[24] Y. Ding, N. Gravish, D.I. Goldman, Physical Review Letters 106, 028001 (2011)

[25] R.D. Maladen, Y. Ding, P.B. Umbanhowar, A. Kamor, D.I. Goldman, Journal of The Royal Society Interface 8, 1332 (2011)

[26] R.M. Nedderman, Statics and kinematics of granular materials (Cambridge University Press, 2005)

[27] S. Dunatunga, K. Kamrin, Journal of Fluid Mechanics 779, 483 (2015)

[28] H. Askari, K. Kamrin, Nature Materials 15, 1274 (2016)

[29] D.L. Henann, K. Kamrin, Physical Review Letters 113, 178001 (2014)

[30] W. Prager, D.C. Drucker, Quarterly Journal of Applied Mathematics 10:2, 157 (1952)

[31] J. Jaky, Journal of the Society of Hungarian Architects and Engineers 78, 355 (1944)

[32] K. Krishnaraj, P.R. Nott, Nature Communications 7 (2016)

[33] L.E. Silbert, D. Ertas, G.S. Grest, T.C. Halsey, D. Levine, S.J. Plimpton, Phys. Rev. E. 64, 051302 (2001)

[34] J.A. Dijksman, G.H. Wortel, L.T. van Dellen, O. Dauchot, M. van Hecke, Physical Review Letters 107, 108303 (2011)

[35] P. Jop, Y. Forterre, O. Pouliquen, Journal of Fluid Mechanics 541, 167 (2005)

[36] D. Fenistein, M. van Hecke, Nature 425, 256 (2003)

[37] D.M. Mueth, Physical Review E 67, 011304 (2003)

[38] O. Pouliquen, Physics of Fluids 11, 542 (1999)

[39] K. Reddy, Y. Forterre, O. Pouliquen, Physical Review Letters 106, 108301 (2011)

[40] E. Wandersman, M. van Hecke, EPL (Europhysics Letters) 105, 24002 (2014)

[41] K. Nichol, A. Zanin, R. Bastien, E. Wandersman, M. Van Hecke, Physical Review Letters 104, 078302 (2010)

[42] M.E. Gurtin, E. Fried, L. Anand, The Mechanics and Thermodynamics of Continua (Cambridge, 2010)

[43] C. Lun, S. Savage, D. Jeffrey, N. Chepurniy, Journal of fluid mechanics 140, 223 (1984)

[44] Y. Jiang, M. Liu, Granular Matter 11, 139 (2009)

[45] I.S. Aranson, L.S. Tsimring, Physical Review E 64, 020301 (2001)

[46] J. Goyon, A. Colin, G. Ovarlez, A. Ajdari, L. Bocquet, Nature 454, 84 (2008)

[47] L. Bocquet, A. Colin, A. Ajdari, Physical Review Letters 103, 036001 (2009) 
[48] D.L. Henann, K. Kamrin, International Journal of Plasticity 60, 145 (2014)

[49] Q. Zhang, K. Kamrin, Physical Review Letters 118, 058001 (2017)

[50] D.L. Henann, K. Kamrin, International Journal for Numerical Methods in Engineering (2016)

[51] P. Jop, Physical Review E 77, 032301 (2008)

[52] T. Unger, J. Török, J. Kertéz, D.E. Wolf, Physical Review Letters 92, 214301 (2004)

[53] S. Savage, M. Sayed, Journal of Fluid Mechanics 142, 391 (1984)
[54] R.D. Maladen, Y. Ding, C. Li, D.I. Goldman, Science 325, 314 (2009)

[55] B. Rodenborn, C.H. Chen, H.L. Swinney, B. Liu, H. Zhang, Proceedings of the National Academy of Sciences 110, E338 (2013)

[56] J. Gray, G. Hancock, Journal of Experimental Biology 32, 802 (1955)

[57] J. Slonaker, D.C. Motley, C. Senatore, K. Iagnemma, K. Kamrin, arXiv preprint arXiv:1604.02490 (2016) 